\title{
La «crise du Chambon », de nouveaux liens entre territoire, institutions, populations et sciences
}

\author{
Frédéric Bally ${ }^{1}$, Marine Gabillet ${ }^{2}$, Denis Laforgue ${ }^{1, *}$, Sandra Lavorel ${ }^{3}$ et Véronique Peyrache-Gadeau ${ }^{4}$ \\ ${ }^{1}$ Sociologie, Université Savoie-Mont-Blanc, LLSETI, Chambéry, France \\ 2 Sociologie, Université Grenoble-Alpes, INRAE, Grenoble, France \\ ${ }^{3}$ Écologie, CNRS, UMR5553 LECA, Grenoble, France \\ ${ }^{4}$ Socioéconomie, Université Savoie-Mont-Blanc, CNRS, UMR5204 EDYTEM, Chambéry, France
}

Reçu le 7 mars 2018. Accepté le 12 septembre 2019

Dans le département des Hautes-Alpes, la fermeture d'une route pendant deux ans a amené les habitants d'une zone enclavée à reconsidérer l'avenir de leur territoire et à transformer cette situation de crise en opportunité d'innovations. Les solutions aux vulnérabilités produites sont loin de converger, entre celles conçues par les spécialistes administratifs ou techniques et celles des habitants eux-mêmes, qui divergent dans leurs choix. Quel peut être alors le rôle d'une équipe de chercheurs investie dans ce territoire pour faciliter l'émergence, au-delà des tensions, des germes de la réinvention du devenir du territoire?

La Rédaction

Résumé - En 2015, un danger d'éboulement de la montagne conduit à couper la route départementale 1091 entre la Haute Romanche et Grenoble. Cette coupure provoque une crise sociale et économique à La Grave et Villar-d'Arêne, deux communes desservies par cette route ; crise qui perdure malgré le rétablissement de la circulation. Cet article propose une analyse qualitative du processus de vulnérabilisation du territoire de Haute Romanche au prisme des interférences, des tensions et des complémentarités qui ont émergé entre la dynamique propre de l'aléa, les processus de décision politique, les expériences (subjectives et pratiques) des populations locales et les enquêtes des chercheurs en sciences sociales et de l'environnement.

Mots-clés : territoire / risques naturels / gouvernance / recherche-action / zone marginale de montagne

\begin{abstract}
Studying the "Chambon crisis " to think the relationship between territories, institutions, populations and sciences. The closing by a landslide risk of the road RD1091, linking the Haute Romanche valley to Grenoble on 10 April 2015, initiated a social and economic crisis with strong impacts on the Villar-d'Arêne and La Grave municipalities. This crisis has persisted even after the reopening of the tunnel and the building of an emergency road. Tourism abruptly stopped with the closing, an acute problem for a territory whose economy relies primarily on this activity: residents lost their capacity to move freely and difficulties to reach hospitals, family and services appeared rapidly. Residents found it difficult to make their situation visible to local institutions: these reacted rapidly from their point of view, but the difference between lived experiences and knowledge, representation of the crisis has created tensions, misinterpretation and conflicts. This paper proposes a qualitative analysis of the process of vulnerability development for the Haute Romanche territory through tensions, interactions and complementarities that emerged between the incident, the political decision, lived experiences (practical and subjective) of the local population and the ongoing work of social science and ecology researchers.
\end{abstract}

Keywords: territory / natural risks / governance / science-action research / marginal mountain area

$\overline{\text { *Auteur correspondant }}$ : denis. laforgue@univ-smb.fr 


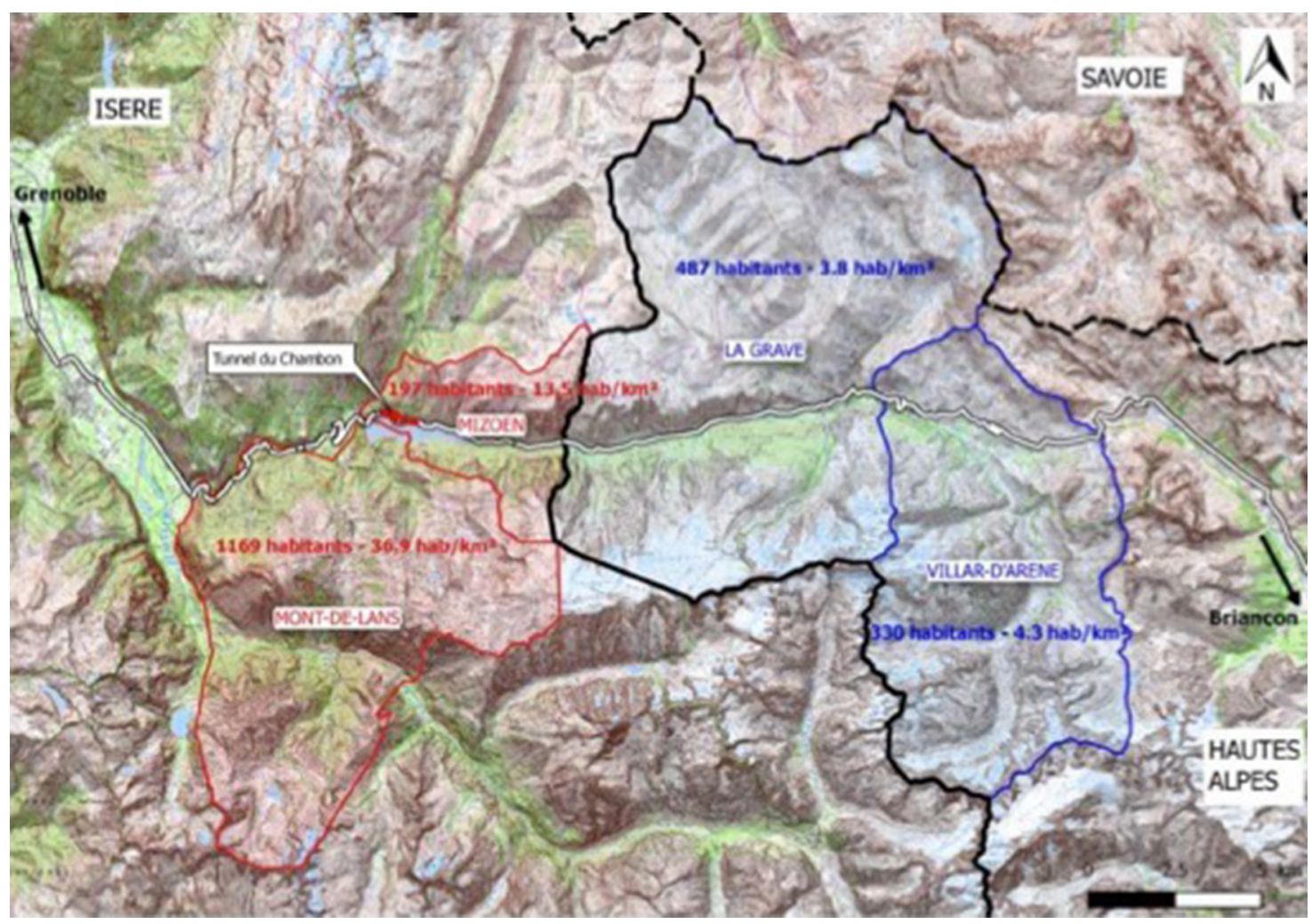

Fig. 1. Carte des communes situées sur le territoire d'étude (source: Cerema Centre-Est; S. Ponen, 2018).

Le 10 avril 2015, la route départementale 1091 entre Bourg d'Oisans et Briançon, qui constitue l'artère de communication de la Haute Romanche, a été fermée à la circulation suite à un accroissement de l'instabilité géologique au niveau du tunnel du Chambon ${ }^{1}$. Après trois mois d'incertitude sur les caractéristiques du glissement de terrain et sur les travaux à engager, la décision a été prise mimai 2015 de fermer la route pour une durée indéterminée. Une route dite « de secours », au trafic limité, a été construite et ouverte à la circulation en novembre 2015 (Fig. 1). C'est à la fin de l'année 2017 que la construction d'un nouveau tunnel a permis la réouverture définitive de la route principale. Pendant toute cette période de coupure de la route, les habitants ont expérimenté divers moyens de transport: navettes lacustres (ensuite stoppées car dangereuses face au risque de glissement de terrain dans le lac du Chambon), sentiers pédestres de montagne (ensuite interdits du fait de l'instabilité de la pente), héliportage (coûteux et donc nécessairement limité dans le temps). Le tableau 1 restitue la chronologie détaillée de ces événements.

\footnotetext{
${ }^{1}$ Cette recherche a fait l'objet d'un premier soutien financier du LABEX Innovation et territoires de montagne (ITEM) [ANR-10-LABX-50-01] dans le cadre du programme «Investissements d'avenir» géré par l'Agence nationale de la recherche (ANR) dans le cadre de l'appel à projets pédagogiques 2015-16 puis en tant que projet exploratoire en 2016 ainsi que d'un soutien par la Zone Atelier Alpes pour l'année 2016 (appel à projet annuel).
}

La gestion de l'aléa naturel et les modalités de réponses qui en ont découlé ont eu des conséquences économiques et sociales considérables pour les habitants des communes de La Grave et de Villar-d'Arêne, directement impactées par la fermeture de la route. Nous employons la notion de « crise» pour désigner la vulnérabilisation durable de la population locale qui s'en est suivie. Nous définissons la vulnérabilité comme un affaiblissement vécu sur le mode de la perte de ressources (financières, symboliques, relationnelles), de la neutralisation de capacités (l'environnement ne permet plus, ne fournit plus les prises pour produire et reproduire un certain nombre de routines) ou de la désorganisation de l'expérience quotidienne (ce qui affecte la sécurité fondamentale des individus, c'est-à-dire leur attitude de confiance dans la continuité du monde et de leur existence).

Un mois après la coupure de la route, certains habitants se constituent en collectif pour défendre les intérêts des populations affectées, organiser des groupes de parole et de concertation pour les réunions publiques. Ce collectif d'habitants et d'acteurs économiques du territoire organise plusieurs mobilisations pour réclamer une nouvelle route. Notre recherche s'inscrit dans ce contexte. Elle a été menée en étroite collaboration avec ces acteurs de la Haute Romanche (principalement des communes de La Grave et de Villar-d'Arêne), d'octobre 2015 à mars 2017.

Pour les chercheurs, la «crise du Chambon» est l'occasion d'une analyse en situation des processus de 
Tab. 1. Chronologie du départ de la crise, de la coupure de la RD 1091 à la création de route de secours provisoire, jusqu'à la réouverture de la RD (réalisation: M. Gabillet d'après S. Ponen, 2018 et les données secondaires récoltées sur le terrain).

10 avril 2015

27 avril 2015

11 mai 2015

13 mai 2015

21 mai 2015

12 juin 2015

23 juin 2015

15 juillet 2015

24 juillet 2015

25 juillet 2015

18 août 2015

3 septembre 2015

24 novembre 2015

9 décembre 2015

15 décembre 2017
Fermeture de l'accès au tunnel du Chambon, coupure de la RD 1091

Mise en place de navettes lacustres

Première réunion publique d'information

Création du collectif Chambon

Manifestation à Grenoble

Mobilisation symbolique des villageois pour réclamer une nouvelle route

Décision de construction d'une route de secours actée/glissement pan de montagne prévu le 4 juillet

Interruption des navettes lacustres et mise en place de navettes aériennes

Venue de Manuel Valls + réunion publique présentation projet piste de secours en rive gauche

Mobilisation collectif du Chambon sur sentier des Aymes pendant le Tour de France

Relative stabilisation glissement terrain, reprise des navettes lacustres

L'Isère opte pour le percement d'un tunnel de dérivation

Ouverture de la route de secours d'intérêt local

L'Isère engage la réalisation des travaux d'un tunnel de dérivation

Réouverture définitive de la RD vulnérabilisation, d'adaptation et d'innovation en territoire de montagne. Les chercheurs impliqués portent des objectifs indépendants et collaboratifs. Il s'agit, d'une part, d'analyser la «vulnérabilité du territoire» (Woloszyn et Quenault, 2013), révélée par la crise, dans ses formes sociales, individuelles, temporelles et spatiales. D'autre part, l'étude des dynamiques socioécologiques du territoire consiste en une "prospective participative» des trajectoires possibles du territoire (Lamarque et al., 2013).

De leur côté, les habitants ont des attentes envers les chercheurs. Ils sont pour eux un acteur externe, «neutre», à qui montrer leurs difficultés pour les légitimer aux yeux des décideurs départementaux et régionaux. Ils espèrent ainsi la reconnaissance de leur expérience. Pour les chercheurs, cette imbrication de la recherche dans l'actualité d'une crise interroge la place de la science dans la société en temps de crise.

Cet article expose les différents récits de la crise par les acteurs impliqués. Il décrit, d'une part, la manière dont les habitants, les experts et les élus vivent les conséquences et les aléas qui suivent la fermeture de la route et, d'autre part, les processus par lesquels ces modes d'expérience variés des événements participent de la crise et de son évolution. L'article interroge aussi la place du chercheur dans le processus d'adaptation et de résilience (Timmerman, 1981) du territoire et de ses habitants.

\section{Méthodologie}

La crise a été appréhendée dans sa dimension économique, sociale, temporelle et spatiale (PeyracheGadeau, 2007), au prisme des vulnérabilités territoriales. Pour cela, l'équipe de recherche s'est saisie d'une méthode qualitative avec des observations in situ, des entretiens avec des habitants et des acteurs en responsabilité ( 40 entretiens au total, avec 3 types d'acteurs, habitants, élus, experts ${ }^{2}$ ) et la collecte de données secondaires (questionnaire réalisé par le collectif d'habitants, enquête publique consultable en mairie où les habitants ont pu s'exprimer de manière anonyme...) sur la période du début de l'hiver 2016 jusqu'à la fin du printemps 2017. Cette étude qualitative, particulièrement adaptée pour comprendre la situation du terrain, le vécu des acteurs (Yin, 2014), offre des données riches (Cuervo-Cazurra et al., 2016). Tous les entretiens ont été retranscrits (certains ont été relatés dans un compte rendu) et ont fait l'objet d'un codage sous le logiciel libre RQDA, avec application d'une grille de code discutée et amendée au fil de l'analyse. La recherche a aussi reposé sur le croisement des regards des chercheurs et des acteurs concernés qui souhaitaient être sujets de la recherche (Les chercheurs ignorants, 2015) et non objets d'enquête et ce, par le biais de réunions, de discussions collectives et de concertation, au fil de l'eau, sur les résultats de la recherche: trois réunions avec des membres du collectif et des habitants, dont une réunion finale de présentation des résultats et de discussion autour de l'évolution de la situation, ont ainsi été organisées.

Ce dispositif d'enquête ethnographique a permis d'appréhender les caractéristiques des vulnérabilités perçues et vécues par les populations et décrites par les institutions qui évaluent les dommages et les réparations suite à la crise. Mais la vulnérabilité territoriale inclut la prise en compte du contexte antérieur à la crise, les conditions de formation du risque, les

\footnotetext{
${ }^{2}$ Des experts, privés ou publics, ont été mobilisés pour travailler sur la résolution de la crise via la construction de la route de secours.
} 
réponses et les adaptations. De manière plus large, autour de la « crise », cette recherche observe les modifications du rapport d'une société montagnarde à son environnement. Dans cette perspective, le croisement de regards disciplinaires (sciences de l'environnement/SHS) s'est révélé pertinent pour décrire les dimensions sociales, économiques et environnementales du changement. Les différents récits et expériences temporelles de l'aléa contribuent à la compréhension de l'évolution de cette crise.

\section{Une tragique vulnérabilisation économique et sociale du territoire}

Situé à la limite des régions Rhône-Alpes et Provence-Alpes-Côte d'Azur et entre les départements de l'Isère et des Hautes-Alpes, le territoire de Haute Romanche est généralement présenté comme aux marges, aux confins, sans orientation précise quant à son appartenance historique, géographique et politique. Les communes impactées par la crise, Villar-d'Arêne et La Grave, sont situées dans le département des HautesAlpes, mais à une faible distance du département de l'Isère et de Grenoble, tout proches, grâce à la route ${ }^{3}$. Habitants, acteurs politiques et acteurs économiques héritent donc d'une distance à leur préfecture, Gap, plus longue que l'accès à Grenoble, élément qui va jouer un rôle dans la dynamique de la crise.

L'économie de la Haute Romanche repose principalement sur le tourisme. Une fragilisation touristique latente, constatée depuis quelques années, a éclaté lors de la fermeture de la route départementale. L'isolement de La Grave et de Villar-d'Arêne durant de longs mois a écarté les touristes. L'étude réalisée pour la chambre de commerce et d'industrie des Hautes-Alpes (Cibles \& Stratégies, 2016) montre que presque un quart des entreprises de Haute Romanche ont des activités d'hébergement ou de restauration. Cette offre touristique est proportionnellement plus forte que pour le reste du Briançonnais. Selon cette étude, sur 43 commerces à La Grave, il existe 23 cafés, hôtels, restaurants, soit plus de la moitié des commerces de Haute Romanche hors hébergements touristiques, chambres d'hôtes, gîtes et résidences touristiques.

Suite à la fermeture du tunnel et de la route départementale en avril 2015, une première onde de crise a durement affecté la saison estivale. La saison hivernale 2016 a été, elle aussi, faible. La même enquête

\footnotetext{
${ }^{3}$ On trouve mention d'un choix fait par ces deux communes d'un rattachement volontaire aux Hautes-Alpes, en 1789 à la création du département, pour espérer bénéficier du statut fiscal et politique des «Escartons du Briançonnais » (source : https:// fr.wikipedia.org/wiki/République_des_Escartons).
}

(Cibles \& Stratégies, 2016) auprès des commerçants et artisans/acteurs économiques de Haute Romanche pointe une évolution négative du chiffre d'affaires des professionnels ( $-32 \%$ entre avril 2015 et avril 2016) avec $94 \%$ des entreprises affichant un chiffre d'affaires en baisse. L'activité artisanale reste moins touchée que les commerces. Cette tendance résulte conjointement de la baisse de fréquentation du territoire par les touristes de passage durant la saison estivale et de la baisse de la fréquentation des stations de ski en saison hivernale. «Enfin voilà, économiquement c'est difficile pour ceux qui ont des charges à payer et qui n'y arrivent pas, il n'y a rien qui rentre et ça donne un aspect assez désert. On avait aussi dans cette commission un suivi de tous les comptages routiers, donc on a bien vu, de 11000 véhicules par jour, on est passé à $2000 \ldots »$ (Cécile, experte, CCI, 8-12-16). À cette perte brutale de clientèle s'ajoute l'incertitude sur le renouvellement de la délégation de service public du téléphérique de la station de La Grave (vallons de la Meije).

Par ailleurs, les aides publiques proposées suite à l'évaluation rapide des difficultés économiques dépendent des dispositifs existants face à l'aléa et semblent trop ponctuelles et inadaptées aux problèmes du territoire et de ses habitants. Ainsi, l'arrêté de catastrophe naturelle a été signé par le Premier ministre Manuel Valls le 31 juillet 2015 afin que les acteurs socioprofessionnels assurés puissent activer la clause de perte d'exploitation. Mais, un an après la fermeture de la route, le dispositif ne permettait pas d'intégrer les pertes qui couraient toujours. Une vulnérabilité financière, associée au coût des procédures de report de paiements et des crédits de soutien, s'est ajoutée aux difficultés sociales et économiques de la crise.

« Nous les commerçants, on a (été) touché(s) à tous les niveaux là. Moral et financier. À tel point que nous, tu vois... on a vu que la saison d'hiver 2015-2016 serait catastrophique [...] Après on nous a embêtés pour faire des dossiers de subvention, interminables pour toucher que dalle, et puis ça ne change rien. Arrêté de catastrophe naturelle, moi je suis encore en train de me battre avec l'assurance, ça fait combien? Un an et demi» (Louis, commerçant, La Grave, 11-10-2016).

Les entretiens indiquent que la vulnérabilisation matérielle et économique a affecté les relations sociales des individus et jusqu'à leurs liens familiaux et leur capacité d'autonomie (Bally et al., 2017). Les entretiens et les questionnaires auprès des deux mairies indiquent des liens directs entre la fermeture de la route et l'irruption de vulnérabilités dans la vie quotidienne des habitants. Il s'agit de pertes de ressources financières : chute du chiffre d'affaires, pertes de salaires, coûts de certaines adaptations (achat ou location de voiture, de matériel, d'un logement, paiement de frais de garde, coût de trajets rallongés). Ce sont aussi des pertes de temps : trajets rallongés pour des raisons professionnelles, 
familiales, de santé, scolaires, de loisir. On observe aussi une perte des relations significatives pour les individus: relations à la famille, bien sûr, liens de sociabilité, mais aussi liens de nature professionnelle (employeurs, salariés, clients) pour certains, et perte de relations à différents services publics (santé, école) ou avec l'espace public (journaux...). Ces différentes pertes ont fragilisé la vie quotidienne de plusieurs individus dans ses dimensions matérielles, bien sûr, à travers une perte relative ou totale des supports matériels de l'autonomie et de l'identité (Sainsaulieu, 1998), mais aussi dans ses dimensions immatérielles, plus difficiles à évaluer et à étayer par les dispositifs publics de gestion du risque naturel. Les réponses à l'enquête publique menée sur les communes de Villar-d'Arêne et de La Grave évoquent, par exemple, «l'impossibilité de recevoir enfants et petits-enfants », «la baisse des visites familiales », les «arrêts de travail à demander »...

Ces différentes pertes affectent les individus sous la forme de «passions tristes » (Deleuze, 2003) qui peuvent s'exprimer de diverses façons. Il peut s'agir d'une peur de l'isolement liée à la perte de temps pour atteindre différents biens, services, institutions (scolaires, de soins, de santé...): «Les médecins spécialistes sont tous sur Grenoble: ophtalmos, cardiologues » (Joseline, habitante, La Grave, 26-01-2016). Les enquêtés soulignent aussi les difficultés à s'adapter à l'isolement: «T'imagines en France, un endroit où t'as pas de tabac et pas de journaux! Mais je crois que... mais je crois que personne ne peut l'imaginer! Et qu'on n'a toujours pas de navette de bus!» (Claire, habitante, Villar-d'Arêne, 11-102016). Difficultés renouvelées par l'interdiction de passage par les sentiers longeant le lac ou passant par la pente. Il est aussi fait état de craintes associées aux propositions successives pour passer sans tunnel, soit via des navettes lacustres (embarcations précaires, équipements de ponton non sécurisés), soit par les airs (héliportage) : «Même avec le bateau, on avait l'impression d'être des migrants, dans des embarcations qui n'étaient pas prévues pour» (Éloöse, habitante, Villard'Arêne, 27-01-2016). Plusieurs enquêtés reconnaissent avoir développé une sensibilité accrue aux risques locaux (danger de la route de secours ouverte à l'automne et dont l'usage a été limité aux résidents de la Haute Romanche précisément pour raisons d'instabilité des abords et d'étroitesse de l'accès) : «La descente, elle est... il y a des pierres qui tombent un peu de partout, où la route elle est quand même très tortueuse et tout. Le Lautaret, bon il y a quelques coups de tourmente dans l'hiver où effectivement ça pose problème» (Philibert, habitant, Villard'Arêne, 10-10-2016).

L'expérience quotidienne de certaines personnes est dominée par un sentiment diffus de désorganisation ou de désordre s'exprimant à un niveau individuel par de la fatigue, des dépressions, des angoisses, de l'irritabilité, de la colère, un sentiment d'injustice vécue, un burn out, un sentiment de non-maîtrise de sa vie: «Enfin on a été en colère pendant six mois, résignés pendant un an et puis là ça... On voit quand même la sortie, ouais ! » (Michelle, habitante, La Grave, 09-12-2016). On observe aussi un sentiment de défiance à l'égard des pouvoirs publics jugés incapables de limiter ou de réparer de manière acceptable ces pertes ou d'écouter les habitants : "C'est ce que $\mathrm{j}$ 'appelle la politique du pourrissement. Il y a un problème, les gens crient, manifestent, et les politiques, c'est ça leur force, c'est qu'ils laissent gueuler les gens, ils reçoivent les coups, ils les acceptent et puis ils savent très bien qu'au bout d'un moment, les gens... tout va s'essouffler » (Claire, habitante, Villar-d'Arêne, 11-102016).

Et, enfin, plusieurs habitants ont le sentiment d'être stigmatisés. Ainsi, à l'échelle du territoire, paradoxalement les réponses institutionnelles à l'enclavement ont pu alimenter la vulnérabilité sociale. Les navettes de bateaux et d'hélicoptères pour transporter les travailleurs en Oisans, la route de secours réservée aux habitants, interdite aux camions, aux cyclistes et aux touristes ont pu, aux yeux des habitants eux-mêmes, stigmatiser ce territoire à un échelon national, voire international.

L'absence de dialogue entre élus locaux et habitants et le manque de mesure des désorganisations successives par les pouvoirs publics ont amplifié l'incertitude autour de la prise en charge du risque naturel. Les moyens ont été raisonnés dans une logique de secours, à partir de dispositifs préétablis, avec un manque d'anticipation et de concertation avec les populations directement concernées et impliquées. Ainsi, près d'un an après la fermeture de la route départementale 1091, l'enquête quantitative réalisée auprès de 164 personnes par le collectif du Chambon en mars 2016 sur les conséquences de la fermeture exprimait ces vulnérabilités sociales toujours très prégnantes: sentiment de précarité renforcé par la perspective de baisse de revenus ; tensions dans la population locale quant aux solutions collectives, souffrances psychologiques (détresse, sentiment d'impuissance, repli sur soi), mais aussi colère et mobilisation collective. Selon cette même enquête, $56,7 \%$ des répondants ressentent les conséquences de la fermeture de la route sur leur santé et leur moral.

L'alerte, la vigilance, les revendications du collectif du Chambon reposent sur cette expérience «tragique» (White, 2009) de la fermeture de la route, cause de perte, de désorganisation et de déclin. Le collectif en tire son sens et sa consistance en termes d'horizon d'action. Aidé par les réunions de concertation avec l'équipe de recherche, il mesure l'épreuve commune, écoute, exprime et institue la controverse en produisant du savoir par les enquêtes auprès des habitants et des professionnels. Il porte un discours et des actions pour « réparer les pertes » et enrayer le déclin en revenant le plus rapidement possible à la situation 
antérieure considérée comme stable et satisfaisante. Il s'agissait, pour ces habitants mobilisés, d'affirmer que «si on veut que le territoire s'en sorte, il faut une mobilisation de tous » pour " prendre des décisions » ou " peser sur les décisions prises par d'autres, mais qui nous concernent» (habitants, réunion de concertation avec le collectif du Chambon, 27-01-16). Pour ce collectif, il faut élaborer des "plans d'action» en déployant une parole publique offensive, stratégique à l'égard de ceux qui sont considérés comme devant apporter une solution, en l'occurrence les services de l'État, de la région et du département. Ils estiment nécessaire la confrontation avec ces institutions par les arguments ou le rapport de force.

\section{Tension entre différents «sens de la crise »}

Même si elle est largement partagée, l'expérience de la «perte» et de la «mobilisation collective» n'est pas la seule façon de vivre et de raconter l'événement... et donc d'y faire face. D'autres récits de la fermeture de la route traversent les acteurs et leur territoire. D'abord, l'opposition entre l'expérience tragique de la crise et le discours technique ressort nettement de nos enquêtes. $\mathrm{Ce}$ discours technique est institutionnel (voir, par exemple, Oger et Ollivier-Yaniv, 2003 ; 2006; Callon et al., 2001), extérieur au territoire et gestionnaire (conseil départemental, conseil régional, préfecture et les structures associées) [Demailly, 1998; Gaulejac, 2009]. Ce récit technique, au sens philosophique du terme (Simondon, 1958), représente la réalité (la fermeture du tunnel et les événements associés) comme un système causal de forces naturelles et sociales. Ces forces sont perçues comme autant de ressources (des moyens) et de contraintes (des obstacles), à maitriser, par des experts extérieurs au système, au problème, à la crise. La définition, l'expérience et la mise en récit technique de la crise réduisent les désordres temporaires du système territorial. Il s'agit de le soumettre à un ordre planifié.

«Nous avons besoin de bâtir une politique sur l'intérêt du secteur de la Haute Romanche, pour pas que Villar-d'Arêne et La Grave soient évités par les touristes à l'avenir. Il est nécessaire de donner de l'unique à ces destinations. On va essayer déjà de créer une zone franche pour les soutenir » (Yves, élu, L'Argentière-laBessée, 28-01-2016).

Dans ce récit technique, l'effondrement du tunnel, le glissement de terrain relèvent de forces naturelles qui perturbent la communication, le passage assurant l'ordre social local: liberté de mouvement, accès à différents services, fonctionnement de différents marchés, etc. Dans l'intérêt général, le rôle des pouvoirs publics est alors de s'appuyer sur des ressources (savoirs experts, moyens matériels) pour modifier l'état (provisoirement désordonné) du système, en réduisant les incertitudes associées (quand fermer le tunnel? quelles solutions alternatives provisoires à la fermeture de la route? où faire passer la route de secours et quelles fonctions lui attribuer? comment soutenir la vie économique locale ?)

Ce registre technique, dont il ne s'agit pas de discuter la cohérence, s'oppose à l'expérience tragique des habitants. Là où le récit technique voit des forces impersonnelles (naturelles, mais aussi économiques [tendances], sociales [masses de populations]), le récit tragique (Rastier, 1999) voit des sujets singuliers, qui ressentent, réfléchissent, mais aussi s'organisent. Le récit technique privilégie les savoirs spécialisés/experts, la distance à l'événement (gage de neutralité et de respect de l'intérêt général) ou encore les preuves objectives et quantifiables (que ce soit en matière de mesure des risques de glissement de terrain ou d'indemnisation des pertes des populations) ; le récit tragique met en avant les usages du territoire-milieu de vie (pour déterminer, par exemple, quand et où construire la route de secours), la proximité intime et l'immersion dans la crise au quotidien (seule voie, selon eux, pour se représenter son ampleur) ou encore le vécu, les affects (par définition non mesurables, non quantifiables). Enfin, le récit technique assure aux élus, représentants politiques et aux experts scientifiques un monopole sur la définition et les décisions; le récit tragique insiste sur le concernement, l'implication et en définitive la responsabilité de chacun et de tous dans les solutions : «On n'est pas plus malin qu'eux, mais on connait le territoire et ses difficultés, ses habitants. On veut leur donner des informations pour qu'ils regardent autre chose que leurs idées, de techniciens » (Éloïse, habitante, Villar-d'Arêne, 27-01-2016).

Cette opposition constante entre deux récits et expériences a alimenté la crise. Elle en a été un élément constitutif, elle transforme la crise technologique (comment restaurer la communication spatiale) en crise politique (défiance à l'égard des institutions, sentiment d'être des citoyens de seconde zone, incompréhension des pouvoirs publics devant certaines actions de contestation) et crise symbolique (lutte pour la définition du rapport du territoire de la Haute Romanche au reste du territoire national). Mais cette opposition entre les habitants locaux et les institutions distantes ne résume pas la diversité des récits en Haute Romanche.

Ainsi, parallèlement à la mobilisation collective incarnée par le collectif du Chambon, on distingue une ténacité silencieuse. Dans ce registre, pour certains habitants, faire face à l'événement et à ses suites n'a pas consisté à exprimer des stratégies collectives (comme si on pouvait résoudre d'un coup un problème par la seule volonté). Ils «tiennent bon» à leur niveau. C'est en situation, au fil des jours, qu'on s'adapte à la fermeture de 
la route, en étant disponible, inventif (intelligence pratique) et solidaire (réactiver les liens entre générations, entre voisins, de parenté même éloignée, etc.) pour saisir le potentiel des situations vécues (être en phase avec la propension des choses et des événements, pour parler comme François Jullien [1992]), sans chercher à les maîtriser, à les diriger. Cela a permis à un certain nombre d'habitants, malgré les problèmes qui s'enchaînaient, d'assurer (à soi et aux proches) une continuité de la relation, du sentier de vie quotidien, de la sécurité ontologique de chacun. «Il y a deux mètres de neige, il faut déneiger, tout le monde déneige la voiture de tout le monde, et puis tout le monde s'aide, alors c'est compliqué, mais à la fois c'est simple» (Claire, habitante, Villar-d'Arêne, 11-10-2016).

Par ailleurs, certains acteurs, en contraste avec un récit tragique, considèrent que le territoire et ses habitants résistent plutôt bien à l'isolement en renouant avec leur nature et leur culture fondamentales, malgré les dimensions négatives (moins de passage, moins de touristes, etc.). Dans ces récits, le territoire est décrit comme un réseau de forces naturelles et culturelles intriquées, voire indissociables. Selon eux, les gens s'adaptent non pas en inventant quelque chose de nouveau, mais plutôt en revenant, en se repliant sur la nature fondamentale de ce territoire : un milieu dur, hostile où les «vrais habitants » (ceux qui sont en phase avec ce milieu) ont l'habitude de composer ; ils sont résistants, sobres (se contentent de peu), habitués à l'isolement et à se débrouiller seuls. Pour eux, l'isolement n'est pas exceptionnel ni grave: l'histoire locale est jalonnée de telles épreuves qui forgent leur caractère et leur authenticité.

« On est une commune rurale, de gens qui vivent là, qui ne sont pas forcément concernés par toute l'effervescence touristique ou médiatique d'aujourd'hui. Ce sont des gens qui vivent, qui ne sont pas forcément préoccupés par tout ça. [...] Tout existe, mais est-ce que les gens ont besoin d'en avoir plus, non » (Charles, élu Villar-d'Arêne, 27-01-16).

Dès lors, dans ce type de récit et d'expérience, l'isolement n'est une crise que pour ceux qui ont oublié ou qui n'ont jamais su ce que c'était de vivre ici : ceux qui se sont installés ou qui sont restés "pour faire de l'argent» grâce aux flux touristiques, ceux qui ont cru pouvoir être tranquilles à la montagne et vivre comme à la ville (logique d'accès permanent à toutes sortes de biens matériels et immatériels). Au final, ce récit développe, en filigrane, une vision assez malthusienne des conséquences de la coupure de la route : pour les détenteurs de cette vision, la crise va opérer comme une sélection économique qui, selon un principe admis de sélection naturelle, produit certes des pertes, mais par là même favorisera la réappréciation de l'offre. La crise va rééquilibrer le territoire après des excès (trop de populations, d'installations, d'argent...) grâce au départ de «tous ceux qui sont là pour de mauvaises raisons » (Charles, élu, Villar-d'Arêne, 27-01-16) et qui ne correspondent pas à l'identité du territoire: ceux qui veulent faire de l'argent, ceux qui n'ont pas renoncé à un mode de vie urbain, qui veulent «jouer sur les deux tableaux ».

Par ailleurs et toujours en contraste avec l'expérience tragique (perte, désorganisation...), émerge aussi un discours où la coupure est certes très dure et suppose une intervention étatique (plus efficace et plus transparente, en particulier en termes de communication politique) et une solidarité locale, mais qui affirme que la Haute Romanche doit surmonter cette épreuve pour se renforcer. Ainsi, pour ces acteurs, la crise réveille le territoire en valorisant ses ressources.

«Nos communes doivent s'associer et mettre en avant leurs ressources, leurs caractéristiques pour créer une offre touristique nouvelle» (Louise, élue, Le Monêtier-les-Bains, 27-01-16).

Pour certains, cela suppose de s'associer à Serre Chevalier ou aux Deux Alpes pour développer un tourisme plus avancé (dépasser un tourisme jugé trop timoré). Pour d'autres, il faut, au contraire, s'appuyer sur le caractère préservé (du tourisme de masse) de la Haute Romanche pour inventer des formes économiques nouvelles (touristiques, mais pas seulement) échappant à un modèle industriel jugé déjà à bout de souffle. «Il faut transformer nos 30 ans de retard en 30 ans d'avance » (Louise, élue, Le Monêtier-les-Bains, 27-01-16). Ces acteurs ont une vision plutôt darwinienne de l'évolution du territoire et des suites de l'événement « coupure de la route». Selon eux, la crise du Chambon sélectionne les plus aptes sur un principe progressiste, ceux qui défendent une vision moderne de l'avenir du territoire, que la crise actuelle va permettre (enfin) de mettre en chantier. Contrairement aux tenants de la vision malthusienne, la crise actuelle ne doit pas, pour ces acteurs, conduire à un retour en arrière (vers des traditions, un éthos consubstantiel au territoire-milieu), mais bien inciter à créer du nouveau (plus adapté à la modernité à laquelle la Haute Romanche ne peut plus tourner le dos) sur les ruines de l'ancien (dont la crise révèle et impose le caractère caduque).

Il faut souligner ici la portée instituante, pour ce territoire de Haute Romanche, de ces différentes manières «de vivre et de faire face» à cet événement (Laforgue, 2015). Premièrement, chacune de ces expériences exprime une façon de se positionner par rapport à différents enjeux constitutifs du territoire et de son avenir, cristallisés par l'isolement (au cœur même des expériences sociales de chaque habitant). Deuxièmement, en interférant les unes avec les autres, ces narrations contradictoires (tragique, technique, malthusienne, etc.) sont constitutives de la crise du Chambon et de ses évolutions et esquissent aussi chacune, dans le même temps, des scénarii de sortie de crise qu'il s'agit 
selon nous de confronter, de faire dialoguer pour que les acteurs arrivent à codéfinir un «monde commun» (Callon et al., 2001) où vivre ensemble.

\section{La crise comme reconfiguration subjective et pratique des liens spatiaux}

Différentes expériences temporelles de la crise existent parmi les récits des acteurs, qui nous ont permis de tracer l'ensemble des événements et des conséquences de l'aléa. En retour, leur intrication définit les évolutions de cette crise. De même, différentes configurations d'expérience matérielle et spatiale constituent cette crise.

La vallée de la Haute Romanche est une entité spatiale et vivre sur ce territoire repose pour chaque habitant sur des relations à des lieux proches et lointains. Ainsi, les habitants soulignent l'importance de ces attachements, que l'on peut qualifier de «liens courts» qui renvoient à l'importance des proximités sensibles, de l'affect, des liens quotidiens entretenus par l'ancrage aux lieux, par choix de vie, de façon assumée, ou par les liens de filiation. Au contraire, les «liens longs» sont associés à la mobilité, au commerce et à la communication à distance, mais aussi à l'accueil, au rapport aux agglomérations envisagées comme des lieux de pouvoir (institutionnels, politiques, économiques...), etc. Ces deux types de liens sont en tension dans des combinatoires mouvantes au fil du quotidien de l'habiter (Leroux, 2008) et de la relation au monde, chacun portant à chaque instant de ses activités une expérience de cette combinaison. Certains habitants sont ainsi attachés au territoire, car y étant nés, et y ont une partie de leur famille, mais développent tout un ensemble de liens plus lointains, ne serait-ce que par le lieu de travail, par exemple.

«Moi je suis née aux terrasses, j’ai toujours vécu ici, j’ai fait mes études sur Grenoble, enfin école à Bourg d'Oisans, Vizille et Grenoble, voilà. Ensuite, et ben j’ai épousé un Portugais et on s'est installés ici parce qu'il travaillait là, et petit à petit il s'est mis à son compte, donc $\mathrm{j}$ 'ai connu le problème Chambon côté entreprise, et moi, ben je travaille à la mairie, avant j'ai fait plein de choses, j'ai travaillé aux Deux Alpes aux remontées mécaniques, au Chazelet, enfin bon j'ai fait plein de petits boulots, et maintenant je suis là à mi-temps » (Lucy, habitante, Villar-d'Arêne, 08-12-16).

La crise du Chambon a affecté ces attachements et ces liens spatiaux et résulte de leur recombinaison. Pour de nombreux habitants, la coupure de la route (en rompant brutalement certains liens longs vers Grenoble) semble accentuer cette tension entre liens courts et liens longs, constitutive du rapport au territoire, jusqu'à en faire un paradoxe vécu. En effet, si les propos mentionnent régulièrement ces liens étroits établis avec le territoire de proximité résidentielle, ces liens à courte distance qui «attachent», sédentarisent, sont aussi, dans le contexte de la fermeture de la route, tout particulièrement perçus comme (dé)limitant la mobilité. L'ambivalence de l'attachement exprime alors une tension entre choix et non-choix d'une dépendance au lieu. Cette tension, perceptible dans les postures individuelles, est aussi décrite comme un tiraillement entre: $1^{\circ}$ d'un côté, un isolement choisi, souhaité, revendiqué, car facteur d'autonomie, au sens où les personnes définissent leur manière propre de vivre sur ce territoire, de manière distincte de la vie urbaine, mais aussi du mode de vie censé caractériser par exemple Serre Chevalier (industrie $\mathrm{du}$ ski, tourisme de masse, etc.); $2^{\circ}$ de l'autre, un isolement subi, imposé par la limitation de l'accès et vu par certains comme l'indice d'une domination ou d'une indifférence étatiques à l'égard de ce territoire des confins, d'où une attente d'inclusion, de rattachement aux territorialités existantes (Bourg d'Oisans, Grenoble, des services publics type EDF ou autres, département, région, préfecture, etc.)

Autre indice de cette tension, on note peu de références à la place de la Haute Romanche dans les territoires institués (politico-administratifs) et dans la recomposition territoriale. La représentation héritée, communément partagée et problématisée à l'occasion de la "crise du Chambon», reste celle d'un territoire historiquement tiraillé entre le Sud (Briançon, les Hautes-Alpes) et le Nord (L'Isère, Bourg d'Oisans, Grenoble). L'appartenance à la communauté de communes du Briançonnais, et celle à l'espace valléen briançonnais sont rarement mobilisées comme territoires de référence par les acteurs ordinaires. Dans les documents de diagnostic (schéma de cohérence territoriale [SCOT] du Briançonnais, par exemple), la vallée de la Haute Romanche occupe pourtant une place singulière dans le Pays du Grand Briançonnais, loin de la zone d'influence de Briançon, et caractérisée comme un pôle touristique avec lequel il faut compter.

Pris dans ce tiraillement, chaque acteur ordinaire du territoire en réduit le caractère problématique en s'orientant sur un pôle ou sur l'autre. Le positionnement antérieur à la crise dans les attachements spatiaux fonde plutôt une expérience tragique liée à l'isolement ou une expérience malthusienne lorsque l'isolement est revendiqué.

Par ailleurs, au fil de la crise, les habitants élaborent deux scénarii de reconfiguration des liens courts et des liens longs : soit une réorganisation provisoire des liens longs, qui réactive dès que possible des liens avec la zone d'influence Grenoble-Lyon: la coupure de la route départementale a d'abord réorganisé les liens longs en les réorientant vers le sud, redéployant les achats vers Briançon et à distance par internet; 1'accès aux services publics (EDF, etc.) est assuré via Briançon ; les transports 
de produits, d'animaux ou de personnes sont détournés vers la Maurienne ou Gap, les liens aux écoles sont réorganisés (collège à Briançon), les relations personnelles (familiales, amicales) et professionnelles à distance sont plus difficiles, voire interrompues. Des acteurs institutionnels proposent la recomposition des liens longs vers Briançon pour une meilleure intégration de la Haute Romanche dans la communauté de communes, voire au-delà vers l'Italie, y compris en convoquant de supposés liens ancestraux. Soit, notonsle, un mode d'expérience spatiale de la crise qui correspond à l'expérience temporelle de la crise sur un registre évolutionniste, «darwinien»: la crise est vue comme une occasion de changement par destruction création (Schumpeter, 1951), épreuve par laquelle le territoire, en s'adaptant, évoluera vers un futur prometteur. «Et puis peut être que comme toute situation aussi, il y a les plus fragiles qui vont malheureusement s'arrêter, et ça va peut-être permettre à d'autres de venir s'installer, ou de rebondir ou... » (Cécile, experte, 08-1216). Mais localement, pour les acteurs ordinaires du territoire, la réorganisation des liens longs est provisoire. L'idée est de maintenir l'existant, de miser sur la réouverture de la route, voire, pour certains, de partir pour sauvegarder des liens longs initiaux: l'accès aux lieux de travail, de formation, de soins. Par ailleurs, la création de la route de secours réinstalle, malgré l'incertitude liée à un accès fragile, les liens longs fonctionnels vers l'Isère.

Le second scénario, adaptatif, privilégie les liens courts pour amortir la crise en s'appuyant sur le système local. La volonté de valoriser la proximité pour combler la défaillance des liens longs a été exprimée très tôt après la coupure. Le renforcement des liens courts, fondés sur les achats de proximité, les services aux personnes, les relations familiales, les liens de travail, les relations de voisinage, est en fait généralement présenté comme la voie privilégiée pour une re-territorialisation des échanges face à l'accroissement des mobilités et à la globalisation des économies et des liens sociaux (Kebir et al., 2017). Ces liens courts sont particulièrement valorisés dans les contextes écotouristiques où la présence des touristes est envisagée comme une source de développement à partir d'une organisation privilégiant la redistribution locale des recettes. La vulnérabilité de la Haute Romanche, de ce point de vue, tient davantage dans sa dépendance aux liens longs (ceux d'un tourisme de passage ou d'un tourisme saisonnier hivernal). Or une solidarité commerciale locale prouve que ces liens courts s'activent au sein de l'économie résidentielle, en se tournant vers la production de produits et de services locaux, en améliorant leur qualité, en travaillant la spécificité de leurs liens aux lieux et en agissant pour la solidarité et le développement de la capacité des sédentaires à organiser leur espace de vie.

\section{Perception d'une recherche-action : la Haute Romanche expérimente des processus d'innovation en montagne}

La crise a donc obligé certains acteurs à innover en matière de modes de vie, de perceptions et d'usages du territoire, de stratégies touristiques, etc. Ce qui ne signifie pas, bien sûr, qu'il n'y a qu'une seule manière d'innover. Et les innovations des uns peuvent être perçues par d'autres comme des « retours en arrière ». Tout d'abord, nos enquêtes montrent les tensions entre trois différentes perspectives sur la crise, et sur les futurs pour le territoire, qui émergent des habitants. Au-delà des acteurs les plus mobilisés, les plus en vue, les plus concernés ou spécialisés sur ces questions, chaque enquêté est travaillé (sous la forme d'un récit et d'une expérience pratique) par une tension singulière entre le passé, le présent et l'avenir de ce territoire. Cela concerne, par exemple, de jeunes adolescents ou des personnes âgées portant des discours sur les «à venir» du territoire tout à fait originaux... et quasiment inaudibles y compris au niveau de la «société des adultes» de la Haute Romanche. La question qui se pose alors est : que faire dans la recherche et pour l'action (collective/politique) de cette épaisseur (on pourrait filer la métaphore du mille-feuilles), de cet enchevêtrement des expériences spatiales et des mises en récit du territoire si on prend conscience qu'il ne s'agit pas (pour le chercheur comme pour le décideur politique) de réduire cette diversité des points de vue à des types ou des tendances. En effet, l'avenir de ce territoire ne peut pas/plus se jouer autour d'un choix entre un tout petit nombre de « scénarii » proposés par des acteurs autorisés, légitimes (au regard d'un statut politique, professionnel, associatif institué).

Cette étude suggère ainsi tout l'intérêt d'une recherche-action collaborative où les habitants sollicitent, interagissent, travaillent avec les chercheurs académiques, alors que souvent ce sont les institutions publiques, les décideurs politiques qui contractent avec les chercheurs pour enquêter sur les populations (leurs points de vue, leurs besoins, leurs satisfactions, etc.). Ce détournement du rapport d'enquête (d'objets, les acteurs ordinaires deviennent sujets) modifie les connaissances et les recommandations produites, pour les rapprocher au mieux du vécu des différents acteurs interrogés. Ainsi, cette recherche souligne, pour les citoyens ordinaires, l'enjeu de reconnaissance du territoire par les institutions publiques. Elle exprime les conditions, fragiles, incertaines de la confiance des habitants de la Haute Romanche en ces institutions. En effet, toute l'enquête s'est structurée autour de l'antagonisme entre les points de vue technico-administratifs sur la crise (son déroulement, la gestion de la crise, etc.) et les expériences vécues des habitants. Cette opposition est constitutive de 
la crise : elle est à l'origine de l'indignation des habitants, de conflits, de souffrances liées à la non-reconnaissance institutionnelle (et qui, par cumul, alourdissent les difficultés du quotidien), mais aussi de l'incompréhension des administrations à l'égard des critiques citoyennes. « On n'a pas à rougir de ce qu'on a fait» (Sébastien, expert mobilisé, SAGE Ingénierie, 08-12-16). « Il y aura toujours des opposants, des gens mécontents, alors comment faire... Même si on fait la meilleure communication du monde, il y aura toujours des gens qui seront toujours... réticents, mécontents » (Ludivine, experte mobilisée, Centre d'études et d'expertise sur les risques, l'environnement, la mobilité et l'aménagement [Cerema], 10-11-16).

Par-delà la compréhension (nécessaire) de cet antagonisme, les chercheurs s'interrogent sur les enseignements de ce cas. Est-ce que l'analyse de cette crise, notamment dans sa dimension temporelle, permet d'entrevoir d'autres options de (co)gestion de futures crises similaires en montagne ? Quel peut être, dans cette perspective, le rôle des institutions de recherche? Notre participation à des dispositifs institutionnels de «retour d'expérience» permettrait une meilleure connaissance de la gestion de ce type de crise.

$\mathrm{Au}$ final, la focale de la recherche sur les expériences vécues de la crise du Chambon par les acteurs ordinaires du territoire (sans responsabilité de gestion de la crise) dessine l'hypothèse selon laquelle, en matière d'innovation et d'adaptation, ce territoire de la Haute Romanche, souvent vu (de l'extérieur, mais aussi par certains de ses acteurs officiels) comme un territoire «à la traîne » (par rapport, par exemple, à des territoires voisins) développe en fait sa propre version de la «surmodernité » (Balandier, 2005). Pour reprendre la distinction opérée par Georges Balandier, la surmodernité s'oppose à la modernité. La modernité, ce sont les sociétés du progrès, les sociétés dont le temps dominant est l'avenir, mais un avenir clair, unique, univoque, unidimensionnel (et donc appauvri). Dans cette conception de la modernité, le territoire voisin qu'est Serre Chevalier peut être considéré comme une société/un territoire moderne, car, de manière dominante, ses acteurs ne pensent son avenir que par le développement (même si c'est avec des aménagements) du tourisme hivernal mondialisé.

Par contraste, la Haute Romanche est surmoderne (et non pas traditionnelle) : son temps de référence n'est pas le passé, c'est ce dont attestent les entretiens avec les habitants. Le temps dominant est le présent, mais un présent épais, gluant, trouble (pour reprendre la métaphore de Donna Haraway [2016]), ni simple ni transparent (pour l'observateur comme pour les acteurs): on se perd dans la multitude des points de vue et des expériences du territoire, qui débordent toutes les typologies sociologiques. Ce présent épais, liquide (Bauman, 2000) se nourrit d'un passé pluriel ( $c f$. la diversité sociale, culturelle, économique, spatiale, générationnelle présente sur le territoire) sans jamais se réduire à la simple répétition de ces passés : ceux qui ont posé leurs valises en Haute Romanche sont venus avec ce qu'ils étaient, mais se sont aussi réinventés en s'installant là et ont réinventé ce territoire. Quant aux « autochtones », là aussi les entretiens montrent que très peu sont dans une logique de reproduction versus de rupture avec les générations précédentes et sont bien davantage dans des formes de réinvention à partir de leurs héritages. Ce présent épais du territoire ouvre sur des avenirs potentiels, multidimensionnels, incertains, à construire... Ce point interroge le rôle des chercheurs dans ces processus : ils ne peuvent plus être des experts, des « sachants » comme dans les sociétés modernes qui croient savoir clairement ce qu'elles veulent et ce qu'elles ne veulent pas, le chercheur pouvant éclairer la marche à suivre. Peut-être, au contraire, le chercheur est-il, désormais, un acteur (parmi d'autres) du territoire, qui, en s'appuyant sur le lien long qui l'attache à ce territoire (au fil de l'enquête), devient une ressource (parmi d'autres) pour la réflexivité des habitants et leurs capacités d'innovation.

\section{Références}

Balandier G., 2005. Le grand dérangement, Paris, Presses universitaires de France.

Bally F., Peyrache-Gadeau V., Gabillet M., Lavorel S., Laforgue D., 2017. Vulnérabilités institutionnelles et résilience territoriale: la construction incertaine d'une trajectoire adaptative en réponse à la «crise du Chambon» en Haute Romanche. Communication au colloque Les défis de développement pour les villes et les régions dans une Europe en mutation, juillet, Athènes (version disponible sur HalSHS, https://hal.archives-ouvertes.fr/halshs-01578912).

Bauman Z., 2000. Liquid modernity, Cambridge (UK), Polity Press.

Callon M., Lascoumes P., Barthe Y., 2001. Agir dans un monde incertain. Essai sur la démocratie technique, Paris, Seuil.

Cibles \& Stratégies, 2016. Étude économique et prospective, Territoire de la Haute Romanche, Chambre de Commerce et d'Industrie des Hautes-Alpes.

Cuervo-Cazurra A., Ganitsky J., Luo Y., Mezias J., 2016. Global strategy and emerging markets. AIB Insights, 16, 4, 3-6, https://documents.aib.msu.edu/publications/insights/ v16n4/v16n4_Article1.pdf.

Deleuze G., 2003 [1 ${ }^{\text {re }}$ éd. 1970]. Spinoza. Philosophie pratique, Paris, Minuit.

Demailly L., 1998. Les métiers relationnels de service public: approche gestionnaire, approche politique, Lien social et Politiques, 40, 17-24, http://dx.doi.org/10.7202/005087ar.

Gaulejac V. de, 2009 [1 ${ }^{\text {re }}$ éd. 2004]. La société malade de la gestion. Idéologie gestionnaire, pouvoir managérial et harcèlement social, Paris, Seuil.

Haraway D.J., 2016. Staying with the trouble. Making kin in the Chthulucene, Durham, Duke University Press. 
Jullien F., 1992. La propension des choses. Pour une histoire de l'efficacité en Chine, Paris, Seuil.

Kebir L., Peyrache-Gadeau V., Crevoisier O., Costa P., 2017. Introduction : sustainability, innovative milieus and territorial development, in Kebir L., Crevoisier O., Costa P., Peyrache-Gadeau V. (Eds), Sustainable innovation and regional development: rethinking innovative milieus, Cheltenham, Edward Elgard, 1-24.

Laforgue D., 2015. Essais de sociologie institutionnaliste, Paris, L'harmattan.

Lamarque P., Artaux A., Barnaud C., Dobremez L., Nettier B., Lavorel S., 2013. Taking into account farmers' decision making to map fine-scale land management adaptation to climate and socio-economic scenarios, Landscape and Urban Planning, 119, 147-157. http://dx.doi.org/10.1016/j. landurbplan.2013.07.012.

Leroux N., 2008. Qu'est-ce qu'habiter? Les enjeux de l'habiter pour la réinsertion, VST-Vie sociale et traitements, 97, 1, 14-25, www.cairn.info/revue-vie-sociale-ettraitements-2008-1-page-14.htm.

Les chercheurs ignorants, 2015. Les recherches-actions collaboratives. Une révolution de la connaissance, Rennes, Presses de l'EHESP.

Oger C., Ollivier-Yaniv C., 2003. Analyse du discours institutionnel et sociologie compréhensive: vers une anthropologie des discours institutionnels, Mots. Les langages $d u$ politique, 71, 125-145, http://dx.doi.org/ 10.4000/mots.8423.

Oger C., Ollivier-Yaniv C., 2006. Conjurer le désordre discursif. Les procédés de «lissage» dans la fabrication $\mathrm{du}$ discours institutionnel, Mots. Les langages du politique, 81, 63-77, http://dx.doi.org/10.4000/mots.675.

Peyrache-Gadeau V., 2007. Approche des dynamiques et des vulnérabilités territoriales: analyse des destinations tou- ristiques à partir des notions de systèmes productifs localisés et de milieux innovateurs, in Lapèze J., Kadiri N. El-, Lamrani N. (Eds), Éléments d'analyse sur le développement territorial: aspects théoriques et empiriques, Paris, L'Harmattan, 131-148.

Ponen S., 2018. Expertise, crise et participation citoyenne. Une co-décision possible? L'expertise dans son système d'acteurs durant la crise du glissement du Chambon (38). Travail de fin d'étude pour le diplôme d'ingénieur de l'École nationale des travaux publics de l'État (ENTPE), Bron/Vaulx-en-Velin, Cerema Centre-Est/ENTPE.

Rastier F., 1999. Action et récit, in Fornel M. de, Quéré L. (Eds), La logique des situations. Nouveaux regards sur l'écologie des activités sociales, Paris, EHESS, 173-198.

Sainsaulieu R., 1998 [1 ${ }^{\text {re }}$ éd. 1977]. L'identité au travail, Paris, Presses de Sciences Po.

Schumpeter J.A., 1951. Capitalisme, socialisme et démocratie, Paris, Payot.

Simondon G., 1958. Du mode d'existence des objets techniques, Paris, Aubier.

Timmerman P., 1981. Vulnerability, resilience and the collapse of society: a review of models and possible climatic applications, Environmental Monograph, 1, Toronto, University of Toronto.

White H., 2009. Poétiques de 1'histoire, Labyrinthe, 33, 21-65, http://dx.doi.org/10.4000/labyrinthe.4029.

Woloszyn P., Quenault B., 2013. Vulnérabilité territoriale et résiliences: résistances et capacités adaptatives face aux aléas climatiques. Communication au $12^{e}$ International Conference of Territorial Intelligence Territorial Intelligence, Socio-Ecological Transition and Resilience of the Territories, 30-31 mai, Besançon-Dijon.

Yin R.K, 2014 (1st ed. 1984). Case study research. Design and methods, Los Angeles, Sage.

Citation de l'article : Bally F., Gabillet M., Laforgue D., Lavorel S., Peyrache-Gadeau V. La «crise du Chambon», de nouveaux liens entre territoire, institutions, populations et sciences. Nat. Sci. Soc. 28, 1, 24-34. 